\title{
Comparative genomic hybridizations reveal absence of large Streptomyces coelicolor genomic islands in Streptomyces lividans Karthik P Jayapal ${ }^{1}$, Wei Lian ${ }^{1,3}$, Frank Glod ${ }^{2,4}$, David H Sherman ${ }^{2}$ and Wei- Shou $\mathrm{Hu}^{* 1}$
}

\author{
Address: ${ }^{1}$ Department of Chemical Engineering and Materials Science, University of Minnesota, 421 Washington Ave. SE., Minneapolis, MN \\ 55455, USA, ${ }^{2}$ Life Sciences Institute, Departments of Medicinal Chemistry, Chemistry, Microbiology \& Immunology, University of Michigan, 210 \\ Washtenaw Ave., Ann Arbor, MI 48109, USA, ${ }^{3}$ Abbott Bioresearch Center, 100 Research Drive, Worcester, MA 01605, USA and ${ }^{4}$ Fonds National \\ de la Recherche, 6 rue Antoine de Saint-Exupéry, L-1017 Kirchberg, Luxembourg \\ Email: Karthik P Jayapal - jayapal@cems.umn.edu; Wei Lian - wei.lian@abbott.com; Frank Glod - Frank.GLOD@fnr.lu; \\ David H Sherman - davidhs@lsi.umich.edu; Wei-Shou Hu* - acre@cems.umn.edu \\ * Corresponding author
}

Published: 10 July 2007

BMC Genomics 2007, 8:229 doi:10.1186/1471-2164-8-229
Received: 2 March 2007

Accepted: 10 July 2007

This article is available from: http://www.biomedcentral.com/I47I-2/64/8/229

(c) 2007 Jayapal et al; licensee BioMed Central Ltd.

This is an Open Access article distributed under the terms of the Creative Commons Attribution License (http://creativecommons.org/licenses/by/2.0), which permits unrestricted use, distribution, and reproduction in any medium, provided the original work is properly cited.

\begin{abstract}
Background: The genomes of Streptomyces coelicolor and Streptomyces lividans bear a considerable degree of synteny. While $S$. coelicolor is the model streptomycete for studying antibiotic synthesis and differentiation, $S$. lividans is almost exclusively considered as the preferred host, among actinomycetes, for cloning and expression of exogenous DNA. We used whole genome microarrays as a comparative genomics tool for identifying the subtle differences between these two chromosomes.
\end{abstract}

Results: We identified five large S. coelicolor genomic islands (larger than $25 \mathrm{~kb}$ ) and 18 smaller islets absent in $S$. lividans chromosome. Many of these regions show anomalous GC bias and codon usage patterns. Six of them are in close vicinity of tRNA genes while nine are flanked with near perfect repeat sequences indicating that these are probable recent evolutionary acquisitions into $S$. coelicolor. Embedded within these segments are at least four DNA methylases and two probable methyl-sensing restriction endonucleases. Comparison with $S$. coelicolor transcriptome and proteome data revealed that some of the missing genes are active during the course of growth and differentiation in S. coelicolor. In particular, a pair of methylmalonyl CoA mutase $(\mathrm{mcm})$ genes involved in polyketide precursor biosynthesis, an acyl-CoA dehydrogenase implicated in timing of actinorhodin synthesis and $b / d B$, a developmentally significant regulator whose mutation causes complete abrogation of antibiotic synthesis belong to this category.

Conclusion: Our findings provide tangible hints for elucidating the genetic basis of important phenotypic differences between these two streptomycetes. Importantly, absence of certain genes in S. lividans identified here could potentially explain the relative ease of DNA transformations and the conditional lack of actinorhodin synthesis in S. lividans.

\section{Background}

Streptomyces spp. include some of the world's most prolific producers of naturally occurring bioactive molecules, many of which are in current therapeutic use [1]. These 
soil-dwelling filamentous bacteria exhibit a remarkably complex life style. Emerging from uni-genomic spores, they colonize the nutrient layer and eventually surface as multi-genomic aerial hyphae, often synthesizing secondary metabolites in the process. The extraordinary diversity of secondary metabolite gene clusters found in these microbes is likely the result of their existence in hostile ecological niches and consequent genomic evolutionary processes including large scale rearrangements, insertions and deletions to cope with these exigencies. The likelihood of these events are further enhanced by the unusual propensity of Streptomyces to undergo spontaneous recombination events especially at chromosome extremities [2,3]. Multiple occurrences of such events over the course of millions of years lead to eventual speciation.

Much of the current knowledge of streptomycetes is based largely on the foundations of genetic and genomic studies conducted in S. coelicolor A3(2) [4]. S. coelicolor is known for its ability to synthesize pigmented metabolites that serve as excellent phenotypic markers in genetic studies. As a model organism, its genome became the first among streptomycetes to be completely sequenced [5]. Nevertheless, S. lividans, a close relative of $S$. coelicolor is almost exclusively considered as the preferred host, among actinomycetes, for heterologous protein expression [6-8]. The primary reasons attributed for this include a significantly relaxed restriction-modification system which enhances exogenous DNA uptake [9] and considerably attenuated endogenous protease activity leading to improved product recovery from S. lividans [7]. Notable examples of biologically active heterologous protein productions in $S$. lividans include proteins of eukaryotic origins like human T-cell receptor CD4 [10], tumor necrosis factor- $\alpha$ [11], human interleukin [12] and salmon calcitonin [13] as well as bacterial proteins like mycobacterial antigens with appropriate glycosylation patterns [14]. Leucotropin ${ }^{\mathrm{TM}}-\mathrm{a}$ recombinant therapeutic agent used in treatment of Hodgkin's disease is commercially produced through $S$. lividans fermentation (Cangene, Winnipeg, Canada).

The 16S rRNA sequences of $S$. coelicolor and $S$. lividans share $>99.5 \%$ identity. Historically, the two species have been distinguished by the inability of the latter to produce the deep blue antibiotic, actinorhodin under many conditions. In addition, S. lividans (1) fails to methylate its own DNA or restrict exogenous methylated DNA (2) possesses diminished extracellular protease activity (3) lacks a mechanism to degrade agar and (4) forms unstable $\phi \mathrm{C} 31$ lysogens [4].

Despite considerable research and economic interests in $S$. lividans and the availability of complete genome sequence of $S$. coelicolor, there have been surprisingly few systematic studies comparing the genome compositions of the two species. Early work by Leblond et al. using pulse-field gel electrophoresis (PFGE) and Southern hybridizations with restriction-fragment linking cosmid probes revealed an essentially similar genomic organization in the two species with identical ordering of the cosmid sequences [15]. More recently, Zhou et al. deduced the absence of a $\sim 90 \mathrm{~kb}$ S. lividans genomic island in S. coelicolor through analysis of a DNA modification deficient $S$. lividans derivative ZX7 [16].

In the post-genomic era, DNA microarrays have emerged as the tool of choice for genome scale comparisons of closely related organisms $[17,18]$. Comparative genomic hybridizations (CGH) using microarrays have already demonstrated their utility in characterization of pathogenicity islands and drug resistance factors in Yersinia pestis [19], Vibrio cholerae [20], Staphylococcus aureus [21] and Mycobacterium tuberculosis [22] among many others. Elsewhere, they have been used to assess genome plasticity and microbial evolution [23]. Among Streptomyces, microarrays have been previously used to detect gross genomic duplications and presence of long terminal repeats at chromosome ends in different strains of S. coelicolor [24]. In this study, we performed a microarray-based whole genome comparison to identify $S$. coelicolor M145 genes absent or divergent in S. lividans TK21. Expression levels of these genes are analyzed and relevant observations are discussed in the context of known phenotypic differences that arose during the recent microevolution of these two species.

\section{Results}

Genome-scale comparison of S. coelicolor and S. lividans We had reported earlier the construction of a wholegenome PCR-product based $S$. coelicolor microarray with probes for more than $95 \%$ of the predicted ORFs [25]. Comparative genomic hybridizations between $S$. coelicolor M145 and S. lividans TK21 using this microarray revealed extensive homology between the two chromosomes. This is apparent from Figure 1a showing the majority of ORFs with relatively similar signal intensity levels from both $S$. coelicolor and $S$. lividans gDNA channels (i.e. $\log _{2}$ of hybridization signal ratio close to zero). The observation concurs well with earlier reports of genome-scale conservation between the two species based on restriction fragment based linkage maps [15]. Genes with $\log _{2}$ hybridization ratios less than the overall mean (of all genes) minus one standard deviation were designated as potentially absent or divergent in S. lividans. Of 7579 S. coelicolor genes probed in our array, we found that only $\sim 8 \%$ fell in this category. Most often, these genes are clustered in localized regions of the chromosome. The clusters themselves, however, are scattered extensively across the entire length of the chromosome, albeit with a definite bias toward the right half. The absence of these clusters in 
S. lividans were confirmed by PCR with primers flanking the missing region (refer to supplementary material for details). Based on the size of these clusters and gene order in S. coelicolor M145, we classified the resident genes as belonging to either one of 5 large genomic islands (GI) $(\geq 25 \mathrm{~kb})$ or 18 smaller islets (Gi) $(<25 \mathrm{~kb})$. These are in addition to the two chromosome ends and about 70 other genes scattered across the chromosome which, despite having low S. lividans gDNA signal, did not satisfy the three-adjacent-gene criteria used here to define a genomic island (see Methods section). The chromosome ends were not termed as genomic islands because large-scale DNA rearrangements are known to occur in the terminal regions leading to difficulties in ascertaining the exact boundaries of islands. Also PCR verification using flanking primers was impossible in such cases.

To assess whether these genomic deletions/insertions are consequences of horizontal gene transfer from distant species, we examined each island for characteristic features that define laterally acquired elements. Hallmarks of laterally acquired element include some or all of the following features - (1) unusual GC bias (2) anomalous codon usage (3) presence in the 3' end of tRNA genes (4) tendency to be flanked by direct repeats and (5) inclusion of mobile genetic elements $[26,27]$. We therefore, sought to evaluate the GC bias and codon usage pattern of genes across the entire genome of $S$. coelicolor. We adopted a windowless cumulative GC plot based on $z^{\prime}$ curve (see Methods section) to evaluate genome-wide GC variation. This approach is more robust, gives higher resolution and has been shown to be useful when the traditional slidingwindow based GC plots fail [28]. A sharp rise in the $z^{\prime}$ value at a localized region in the chromosome implies an unusually AT-rich segment. For assessing codon usage patterns, we calculated the Codon Adaptation Index (CAI), a measure of codon optimality [29], for every ORF with respect to the most frequent codons occurring in S. coelicolor. CAI values range from 0 to 1.0 with higher values indicating more optimal codon usage. Both the cumulative GC plot and CAI plot revealed excellent correlation with regions identified using microarrays (Figure $1 \mathrm{~b}$ and 1c). In particular, 18 out of 23 islands/islets identified from microarray analysis have an average CAI $\leq 0.60-\mathrm{a}$ significant departure from an average of 0.73 for the whole genome. There were, however, some regions with low CAI that were not identified as absent in S. lividans. Although, it is quite possible that these segments might have been laterally acquired, our results suggest that those gene transfer events occurred before the evolutionary divergence of these two species. In addition, six of the 23 regions are located at 3 ' ends of tRNA genes while nine are flanked by near-perfect direct repeat sequences. Some of these repeat sequences had significant homology to the att sites of certain plasmids or phage related elements. For example, the repeat sequences flanking Gi-4, Gi-6, Gi-11 and GI-5 resembled the att sites of actinophage VWB, plasmid pSG1 (S. griseus), pSLP2 (S. lividans) and pMB400 (Bacillus megaterium) respectively. The list of genomic islands and islets identified and their associated features are shown in Table 1. Taken together, these results suggest that many of these segments are recent evolutionary incorporations into the $S$. coelicolor chromosome.

\section{Analysis of $\mathrm{Gls}$ and resident genes}

A total of 625 genes reside within the S. coelicolor genomic islands/islets identified above. They were categorized based on their functional assignments (Table 2). Unsurprisingly, more that two-thirds of laterally acquired elements in S. coelicolor are absent in S. lividans. Mobile genetic elements like integrative plasmids, bacteriophages and transposon related genes constitute this class. Other genes missing in S. lividans are comprised largely of hypothetical and periplasmic/exported proteins although a significant fraction belongs to a diverse set of functional categories shown in Table 2.

GI-1 (22 ORFs; $26 \mathrm{~kb}$ ) consists of a variety of genes including those coding for acetate uptake, amino acid biosynthesis and possible iron uptake systems. Superoxide dismutase - SCO0999 (sodF2), an Fe-dependent antioxidant was previously hypothesized to have been acquired by horizontal gene transfer [30]. SCO0981-83 constitutes an acetate uptake system with a DNA-binding regulator $($ aceR), isocitrate lyase (aceA) and malate synthase (aceB2) genes. Isocitrate lyase and malate synthase are required for a functional glyoxylate pathway which promotes acetate utilization; yet, expression of these genes in S. cinnamonensis did not restore growth of acetate-uptake-deficient mutants on acetate as sole carbon source giving rise to the possibility that some, as yet unknown, factors are missing [31]. SCO0997 and SCO0998 are annotated as ironuptake system proteins - $f t r D$ and $f t r E$ respectively. However, the presence of an upstream gene (SCO0996) homologous to a lipoprotein found in daptomycin biosynthesis cluster has raised questions about their involvement in iron uptake [32]. This is further corroborated by the presence of chromosomally linked genes homologous to SCO0991/92 (a hypothetical protein and a putative cysteine synthase) near the daptomycin cluster of $S$. roseosporus [33]. If true, the presence of these antibiotic biosynthesis linked genes in a genomic island of $S$. coelicolor would be intriguing in view of evidence for horizontal transfer of antibiotic clusters among streptomycetes isolated from soil [34].

GI-2 (70 ORFs; 75 kb) includes ORFs encoding a number of transposases and insertion elements. Interspersed within this region are a putative extracytoplasmic function sigma factor (SCO3450), a methyltransferase 
a.

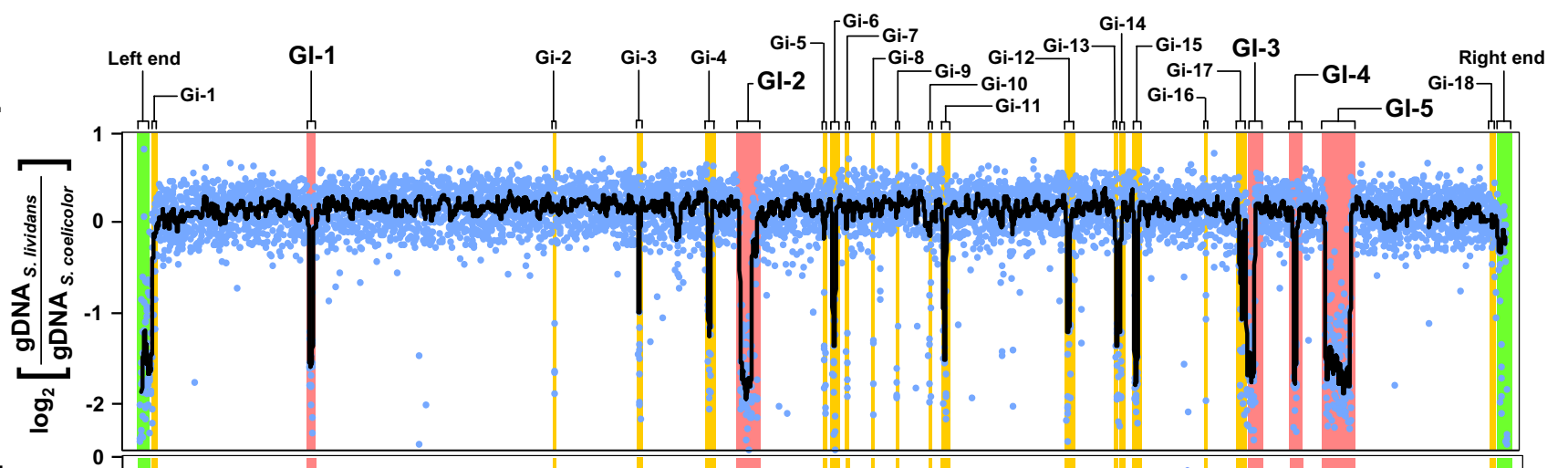

b.

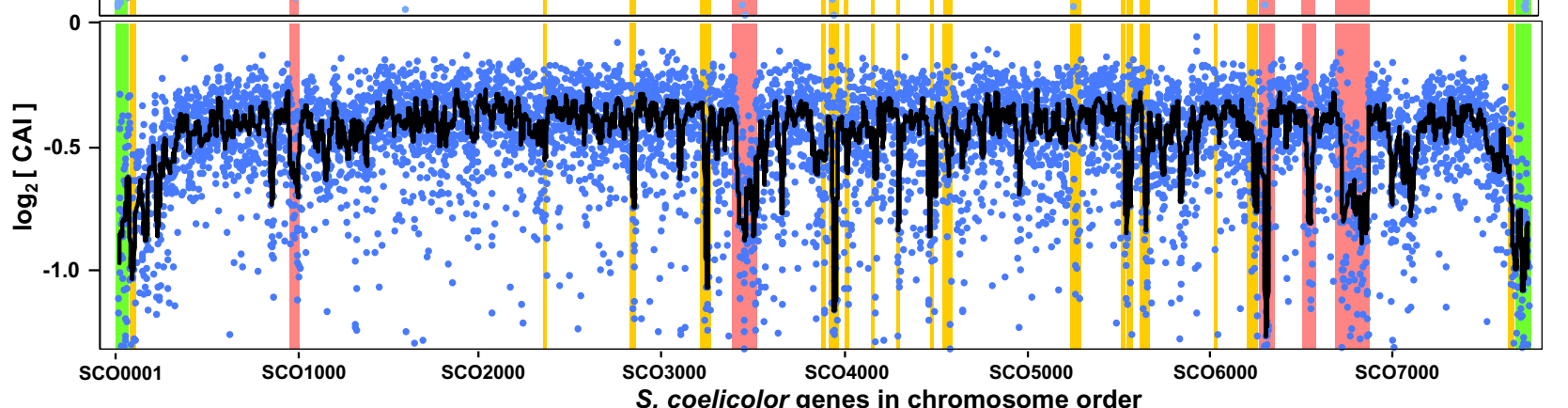

C.

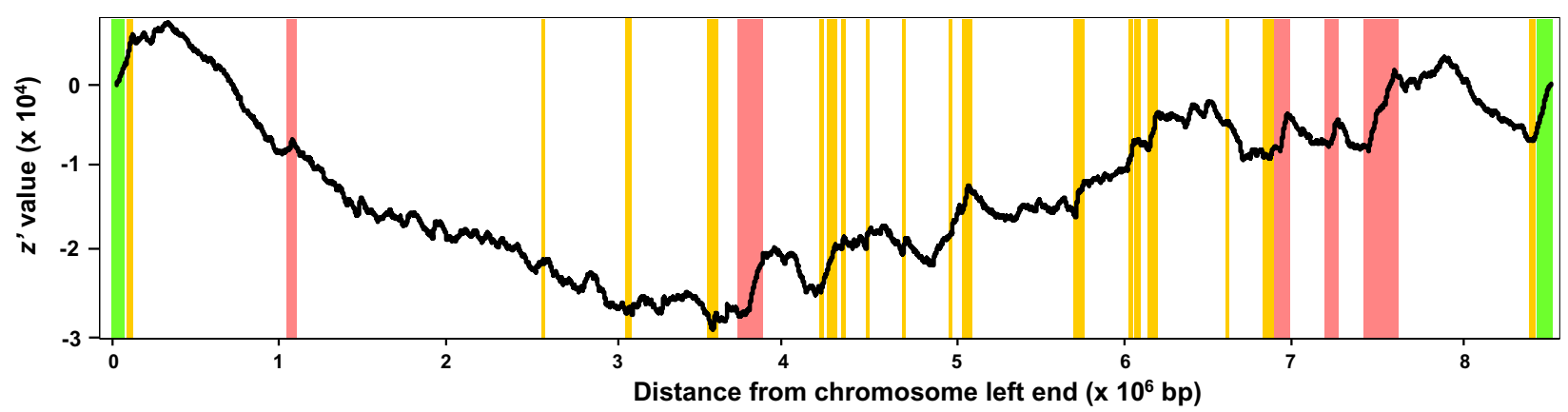

Figure I

Comparative analysis of S. coelicolor MI 45 - S. lividans TK2I chromosomes and genomic features in S. coelicolor. (a) Log 2 hybridization ratio as a function of $S$. coelicolor ORFs. Low values imply absence or divergence of gene in $S$. lividans. Experimental data (blue circles) and smoothened data using median of a 19-gene sliding window (thick line) are shown. Large genomic islands (red), smaller islets (orange) and S. coelicolor specific chromosome end regions (green) identified in this study are marked with filled bars. The sizes of the bars approximate the extent of each island. Gl and Gi numbers corresponding to those in Table I are indicated. Note that the smoothened curve, shown here for ease of visualization, will not show a dip in hybridization ratios for genomic islets with fewer than 10 genes. (b) Logarithm of codon adaptation index (CAI) as a function of S. coelicolor ORFs. Low $\log _{2} \mathrm{CAl}$ implies sub-optimal codon usage. (c) $z^{\prime}$ value plotted as a function of distance from chromosome left end. A sharp increase in $z^{\prime}$ value indicates presence of an AT-rich region. The figure shows that there exists a good correlation between presence of genomic islands, codon usage bias and local GC content.

(SCO3452), a set of putative ABC-type transporters related to spermidine/putrescine transport family (Pot) (SCO3453-56) and an assortment of consecutive genes with various putative metabolic activities (SCO34733506). It is noteworthy here that the only extracellular agarase (dagA, SCO3471) of S. coelicolor maps to this genomic segment. This explains the lack of agarase activity in cultures of S. lividans noted elsewhere [35].

GI-3 (53 ORFs; 57 kb) contains elements of three putative two-component systems (SCO6353/54, SCO6362/ 63/64 and an orphan kinase SCO6369). The region also 
Table I: Characteristics of genomic islands (GIs) and islets (Gis) identified in this study

\begin{tabular}{|c|c|c|c|c|c|c|c|}
\hline Element ${ }^{a}$ & Size (kb) & $\begin{array}{l}\text { Number of } \\
\text { ORFs }\end{array}$ & $\%$ GC Content $b$ & $\begin{array}{l}\text { Average CAI } \\
\text { (Percent genes } \\
\text { with low CAI)c }\end{array}$ & tRNA in Vicinity & $\begin{array}{l}\text { Flanking Direct } \\
\text { Repeats }\end{array}$ & $\begin{array}{l}\text { Mobile Genetic } \\
\text { Elements }\end{array}$ \\
\hline $\begin{array}{l}\text { Chromosome left end; } \\
\text { SCO000I-008I }\end{array}$ & 69.6 & 81 & 69.5 & $0.58(59 \%)$ & - & - & $\begin{array}{c}8 \text { transposon related } \\
\text { genes }\end{array}$ \\
\hline Gi-I; SCO0090-0099 & 6.6 & 10 & 67.1 & $0.56(67 \%)$ & - & - & $\begin{array}{c}6 \text { transposon related } \\
\text { genes }\end{array}$ \\
\hline GI-I; SCO0979-I000 & 25.9 & 22 & 70.7 & $0.67(23 \%)$ & - & - & I phage related integrase \\
\hline Gi-2; SCO238I-2384 & 6.4 & 4 & 68.4 & $0.57(75 \%)$ & - & - & - \\
\hline Gi-3; SCO2862-287I & 8.6 & 10 & 65.9 & $0.54(78 \%)$ & - & - & $\begin{array}{l}\text { I gene similar to phage } \\
\text { replication regulator }\end{array}$ \\
\hline Gi-4; SCO3250-3270 & 14.0 & 21 & 66.2 & $0.54(80 \%)$ & Arg-tRNA CCT & 43 bp perfect repeat & $\begin{array}{l}\text { Mostly plasmid (pSAM2) } \\
\text { related genes }\end{array}$ \\
\hline GI-2; SCO3437-3506 & 75.0 & 70 & 68.7 & $0.60(55 \%)$ & - & $\begin{array}{l}61 \text { bp repeat (6 } \\
\text { mismatches) }\end{array}$ & $\begin{array}{c}5 \text { transposon related } \\
\text { genes }\end{array}$ \\
\hline Gi-5; SCO3929-3937 & 9.2 & 8 & 68.0 & $0.59(50 \%)$ & - & - & $\begin{array}{l}\text { PSAM2 integration site, } \\
\text { some plasmid functions }\end{array}$ \\
\hline Gi-6; SCO3980-3998 & 10.7 & 19 & 65.8 & $0.44(100 \%)$ & Ser-tRNA TGA & $\begin{array}{l}106 \text { bp repeat ( } 8 \\
\text { mismatches) }\end{array}$ & $\begin{array}{l}\text { PSAM2 insertion } \\
\text { element }\end{array}$ \\
\hline Gi-7; SCO4060-4066 & 5.2 & 7 & 66.1 & $0.43(100 \%)$ & Ser-tRNA GGA & $\begin{array}{l}88 \text { bp repeat ( } 4 \\
\text { mismatches) }\end{array}$ & $\begin{array}{c}2 \text { transposon related } \\
\text { genes }\end{array}$ \\
\hline Gi-8; SCO4210-42I3 & 3.8 & 4 & 66.8 & $0.50(75 \%)$ & - & 19 bp perfect repeat & $\begin{array}{l}\text { Some phage related } \\
\text { genes }\end{array}$ \\
\hline Gi-9; SCO4346-4350 & 7.0 & 5 & 66.9 & $0.55(75 \%)$ & - & - & - \\
\hline Gi-10; SCO4533-4537 & 4.3 & 5 & 72.1 & $0.70(0 \%)$ & - & - & - \\
\hline Gi-II; SCO46I5-463I & 15.9 & 17 & 68.3 & $0.62(31 \%)$ & Tyr-tRNA GTA & $\begin{array}{l}\text { II } 2 \text { bp repeat (I } \\
\text { mismatch) }\end{array}$ & Possible SLPI insertion \\
\hline Gi-I2; SCO5327-5350 & 21.3 & 24 & 65.3 & $0.67(55 \%)$ & Arg-tRNA CCG & $\begin{array}{l}\text { I I } 4 \text { bp repeat ( } 4 \\
\text { mismatches) }\end{array}$ & $\begin{array}{l}\text { PSAM2 integration, } \\
\text { plasmid/phage related } \\
\text { genes }\end{array}$ \\
\hline Gi-I3; SCO5605-5620 & 12.9 & 16 & 67.0 & $0.58(67 \%)$ & - & - & All phage related genes \\
\hline Gi-I4; SCO5632-5644 & 10.4 & 13 & 63.8 & $0.44(100 \%)$ & - & $\begin{array}{l}52 \text { bp repeat ( } 5 \\
\text { mismatches) }\end{array}$ & $\begin{array}{l}\text { Some plasmid related } \\
\text { functions }\end{array}$ \\
\hline Gi-I5; SCO57I8-5735 & 21.8 & 18 & 68.1 & $0.56(72 \%)$ & - & - & - \\
\hline Gi-16; SCO6I20-6124 & 2.9 & 5 & 70.0 & $0.67(25 \%)$ & - & - & - \\
\hline Gi-17; SCO63|4-6338 & 23.6 & 23 & 70.2 & $0.59(70 \%)$ & - & - & $\begin{array}{c}2 \text { transposon related } \\
\text { genes }\end{array}$ \\
\hline GI-3; SCO6353-6405 & 56.6 & 53 & 68.6 & $0.56(61 \%)$ & - & - & $\begin{array}{c}4 \text { transposon related } \\
\text { genes }\end{array}$ \\
\hline GI-4; SCO6625-6642 & 30.4 & 18 & 68.6 & $0.56(65 \%)$ & - & - & Few phage related genes \\
\hline GI-5; SCO6806-6953 & 153.3 & 148 & 69.0 & $0.60(53 \%)$ & Pro-tRNA GGG & 44 bp perfect repeat & $\begin{array}{l}\text { I transposon related } \\
\text { gene at end }\end{array}$ \\
\hline Gi-18; SCO7795-7802 & 4.2 & 8 & 65.2 & $0.48(86 \%)$ & - & - & $\begin{array}{c}3 \text { transposon related } \\
\text { genes }\end{array}$ \\
\hline $\begin{array}{l}\text { Chromosome right end; } \\
\text { SCO7827-7845 }\end{array}$ & 20.4 & 19 & 70.1 & $0.55(83 \%)$ & - & - & $\begin{array}{l}\text { I transposon related } \\
\text { gene; }\end{array}$ \\
\hline
\end{tabular}

a Large genomic islands $(\geq 25 \mathrm{~kb})$ are in bold face

b Percentage GC content within each genomic island; compare with $72.1 \%$ average GC content of whole genome

c Compare the average CAl of ORFs within each segment with 0.73 , the average CAl of all genes in S. coelicolor. Percent genes with low CAl is the fraction of genes in each

island with CAI values lower than global mean - standard deviation $(=0.625)$

encompasses ORFs with bacitracin transport permease domains (SCO6356, 6360, 6378). The last 16 ORFs in this island comprise mainly of mobile genetic elements like transposases and recombinases.

GI-4 (18 ORFs; 30 kb) contains a phage growth limitation $(\mathrm{Pgl})$ system. This is a defense mechanism against $\phi$ C31 bacteriophage infections in which an infecting phage undergoes a single burst of attack and the resulting progeny are severely attenuated in subsequent infectious cycles. Two loci, $p g l W X$ and $p g l Y Z$ - both of which map to this genomic locus - are necessary for the $\mathrm{Pgl}^{+}$phenotype. Absence of these genes in S. lividans leads to unstable $\phi \mathrm{C} 31$ lysogens while complementing them with these elements give rise to $\mathrm{Pgl}^{+}$colonies [36].
GI-5 (148 ORFs; 153 kb) is the largest genomic island identified in this study. The size of this cluster implies that it hosts a multitude of genes with varied functions. Importantly, this cluster hosts genes that are involved in various modes of microbial defense. SCO6826/27 encodes a pair of type I modular polyketide synthase genes. In close proximity are two genes (SCO6832/33) coding for subunits of methlymalonyl-CoA mutase - an enzyme that catalyzes conversion of succinyl-CoA to methylmalonylCoA, which serves as building blocks for certain polyketide antibiotic synthesis [37]. Two other genes, SCO6929/30 are similar to those involved in lantibiotic biosynthesis. In addition, SCO6835/36/37 belong to the arsenic resistance family. SCO6809/10 are similar to several multi-drug efflux transporters. Also, a probable acylCoA dehydrogenase (SCO6938) belonging to this island 
Table 2: Functional classification of S. coelicolor MI 45 genes missing or divergent in S. lividans TK2 I

\begin{tabular}{|c|c|c|c|}
\hline Function $^{a}$ & Total Present ${ }^{b}$ & Number Missing/Divergent & Percent Missing/Divergent \\
\hline Cell processes & 800 & 32 & $4 \%$ \\
\hline Macromolecule metabolism & 496 & 20 & $4 \%$ \\
\hline Amino acids biosynthesis & 123 & 3 & $2 \%$ \\
\hline Nucleotide biosynthesis & 30 & 0 & $0 \%$ \\
\hline Ribosomal constituents & 67 & 0 & $0 \%$ \\
\hline Biosynthesis of cofactors and carriers & 118 & 0 & $0 \%$ \\
\hline Central intermediary metabolisms & 111 & 4 & $4 \%$ \\
\hline Degradation of small molecules & 200 & 6 & $3 \%$ \\
\hline Energy metabolism & 189 & I & $1 \%$ \\
\hline Fatty acid and phosphatidic acid biosynthesis & 56 & 4 & $7 \%$ \\
\hline Secondary metabolism & 277 & 3 & $1 \%$ \\
\hline Periplasmic, exported or lipoproteins & 1318 & 84 & $6 \%$ \\
\hline Two-component systems & 165 & 7 & $4 \%$ \\
\hline RNA polymerase core enzyme binding & 88 & 3 & $3 \%$ \\
\hline Regulatory proteins & 673 & 39 & $6 \%$ \\
\hline Protein kinases & 39 & 1 & $3 \%$ \\
\hline Laterally acquired elements & 139 & 95 & $68 \%$ \\
\hline Not classified & 565 & 30 & $5 \%$ \\
\hline Hypothetical proteins & 2371 & 293 & $12 \%$ \\
\hline Total & 7825 & 625 & $8 \%$ \\
\hline
\end{tabular}

${ }^{a}$ protein classification scheme derived from EcoCyc database; downloaded from http://www.sanger.ac.uk/Projects/S coelicolor/scheme.shtml

${ }^{b}$ Total number of proteins present in S. coelicolor chromosome in each category

has been implicated in control of actinorhodin production and timing of sporulation [38]. This observation is particularly interesting considering the extremely low-levels of actinorhodin production [39] and absence (or silencing) of the ram-independent developmental pathway in S. lividans [40]. Furthermore, two putative DNA methylases (SCO6844 and SCO6885) also map to this locus.

The smaller genomic islets (Gi-1 to Gi-18) also contain several potentially interesting genes. Foremost among them is Gi-15. This islet includes the bldB locus (SCO5723) which has been attributed to pleiotropically regulate both antibiotic synthesis and morphological differentiation [41]. Two other genes found in this islet (SCO5722 and SCO5731) encode putative secreted serine proteases. This islet is also unusual in being flanked on one side by a highly expressed $r p s O$ gene rather than a tRNA. Among other smaller islets, Gi-12 contains an adenine-specific DNA methylase (SCO5331, pglS). This gene has been confirmed to interfere with the Pgl system of GI4 and might extend resistance to phages other than $\phi \mathrm{C} 31$ [42]. Gi-10 and Gi-11 each encode a methyl specific restriction endonuclease - SCO4213 (mrr-like) and SCO4631 ( $m c r A$-like) respectively. The rest of the genes in the genomic islands/islets are largely comprised of hypothetical proteins and other genes whose functions are yet to be elucidated.

\section{Comparison with transcriptome and proteome data}

Assuming that these genomic islands have evolved under selective pressure, we asked which, if any, of these genes are actively expressed under normal (laboratory) culture conditions. We chose to analyze mRNA and protein expression data derived from two distinct liquid media one, an R5-based rich medium with yeast extract and the other, a minimal medium supplemented with casaminoacids (SMM). Due to significant differences in their composition, we expected that they will elicit markedly distinctive gene expression patterns. Data for the R5-based cultures were generated using our microarray and LC-MS experiments (Jayapal et al., in preparation) while those for SMM cultures were downloaded from a public repository http://streptobase.org/ or from previously published 2-D gel proteomic analyses $[43,44]$. Since the microarray hybridizations were performed with $S$. coelicolor gDNA reference, the resulting $\log _{2}$ expression ratios $\left(=\log _{2}\right.$ $\left[\mathrm{mRNA}_{S c o e} / \mathrm{gDNA}_{\text {Scoe }}\right]$ ) are an indication of the expression level for each gene [25]. The highest $\log _{2}$ expression ratio observed for a given gene over the range of growth phases and conditions analyzed can be taken as its potential for transcription under laboratory conditions. Figure 2 shows this transcriptome expression data plotted against comparative genomics hybridization data. Genes in the topleft region of the plot (marked in Figure 2) are, in general, designated as absent in S. lividans, yet highly expressed in $S$. coelicolor at least in some conditions. Note that this 
region also includes a few genes with quite low $\log _{2}$ $\left[\mathrm{gDNA}_{\text {Sliv }} / \mathrm{gDNA}_{\text {Scoe }}\right]$ value but were not designated as absent/divergent in S. lividans simply because they did not co-localize as three- or more-gene clusters and hence did not satisfy out absence criteria (see Methods section). Reciprocally, some genes with high $\log _{2}\left[\mathrm{gDNA}_{\text {Sliv }} / \mathrm{gDNA}_{S \text { - }}\right.$ ${ }_{c o e}$ ] were assigned to certain genomic islands because of overwhelming evidence of absence from neighboring genes. Many such genes with considerable homology are transposon/plasmid related elements and are probably present elsewhere in the S. lividans chromosome. 42 genes from various genomic islands exceeded a threshold of $\max \left(\log _{2}\left[\mathrm{mRNA}_{S c o e} / \mathrm{gDNA}_{S c o e}\right]\right)$ value greater than 2.0 indicating appreciably high mRNA expression. 20 of these genes also had an average $\left(\log _{2}\left[\mathrm{mRNA}_{\text {scoe }} / \mathrm{gDNA}_{\text {scoe }}\right]\right)>0$ indicating probable constitutive basal expression (data not shown in Figure). Currently, more than half of those 42 genes (23/42) do not have a reasonably descriptive functional annotation.

The most striking observation from the transcriptome analysis was the high expression of $\sim 30$ contiguous genes (SCO3473-3506) within GI-2 exclusively in SMM cultures. These genes encode a variety of probable carbohydrate metabolism enzymes including putative aldolase, isomerase, dehydrogenase, beta-galactosidase, sugar kinase and sugar permease; the precise context of these functions remain to be elucidated. Nevertheless, such coordinated but conditional transcription of genes within genomic islands leads one to speculate that, in addition to collective horizontal transfer as an island, they are also regulated in an independent and rather concerted fashion in response to varying environmental stimuli. In addition, the extracellular agarase precursor (SCO3471) in GI-2 is also expressed highly only in SMM cultures.

Several genes in GI-1 are also highly expressed. The superoxide dismutase (SCO0999, sodF2) has an average $\left(\log _{2}\right.$ $\left[\mathrm{mRNA}_{\text {Scoe }} / \mathrm{gDNA}_{\text {scoe }}\right]$ ) of over 2.0 in cultures of R5-based medium and was also identified in LC-MS proteomic studies with multiple peptide hits (Jayapal et al., in preparation). However, this gene is not as highly expressed in SMM cultures. On the contrary, adjacent genes SCO0985/ $0993 / 0995$ were identified in the 2-D gel experiments conducted on minimal medium derived samples [43]. Genes in bldB locus (Gi-15) are also expressed at moderate to high levels from microarray data (in both rich and minimal medium). This is corroborated by the identification of some of these proteins (SCO5723/24 and SCO5729) in proteomic experiments. Interestingly, SCO5724/25 possibly codes for a novel secretion system [45]. Such transporters are widely associated with pathogenicity islands in many bacteria [26]. Furthermore, phage resistance proteins of the Pgl system (SCO6627/28/37/38; GI-4) were identified in several proteomic experiments. Notably, one

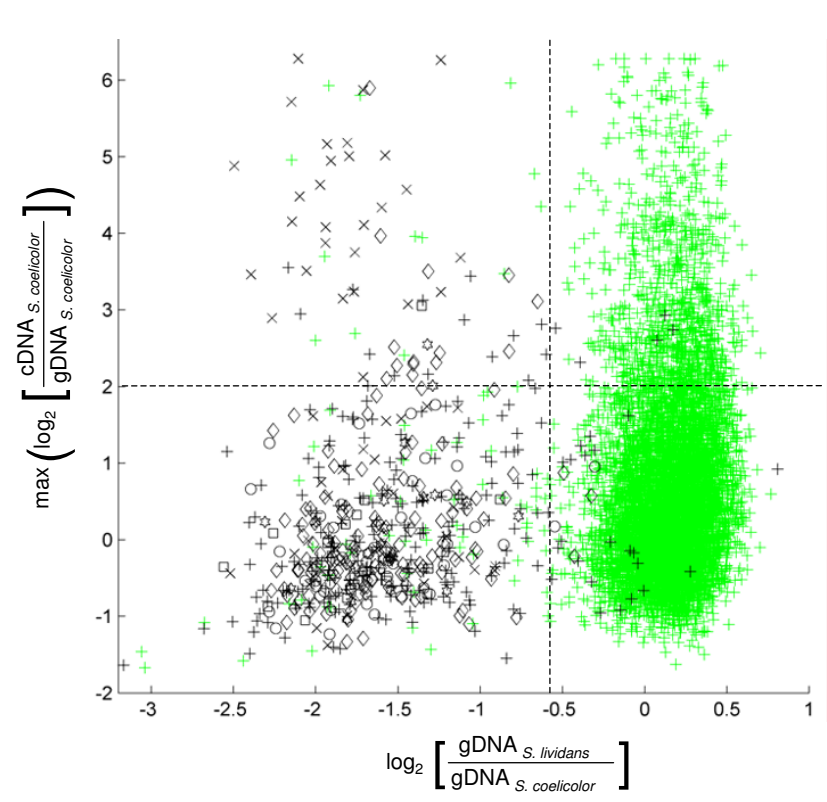

Figure 2

Comparison of $\mathrm{CGH}$ and transcriptome data. Plot of $\log _{2}$ [gDNA $\left._{\text {sliv }} / g D N A_{\text {scoe }}\right]$ (x-axis) vs $\max \left(\log _{2}\left[\mathrm{mRNA}_{\text {Scoe }} / g D N A_{S-}\right.\right.$ coe]) ( $y$-axis). The $y$-axis values correspond to the maximum gene expression levels observed considering all analyzed samples (includes both R5- and SMM). Genes belonging to GI-

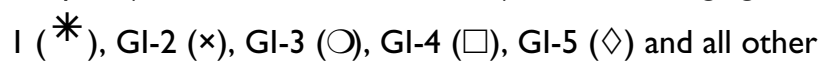
smaller islets (black + ) are shown alongside genes conserved in both S. coelicolor and S. lividans (green + ). Genes in the region left of and above the dotted lines are those that are highly expressed in S. coelicolor but absent in S. lividans. High expression in this case was defined as $\max \left(\log _{2}\left[\mathrm{mRNA}_{\text {Scoe }} l\right.\right.$ gDNA $\left.\left._{\text {Scoe }}\right]\right) \geq 2.0$ which corresponds to the top 14 percentile of gene expression values. The plot shows that a large fraction of genes absent in S. lividans have low expression levels in S. coelicolor. Nevertheless, there are some prominent examples of highly expressed genes within certain S. coelicolor genomic islands. This includes several genes from Gl-2.

of them - SCO6627 (PglX) was identified in multiple forms in the membrane fraction of mycelium undergoing programmed cell death [44]. Likewise, polyketide precursor synthesis enzymes (SCO6832/33, methylmalonylCoA mutase subunits in GI-5) were identified in LC-MS experiments of $\mathrm{R} 5$ medium samples (Jayapal et al., in preparation). 


\section{Discussion \\ S. coelicolor contains large laterally acquired genomic segments}

Our results indicate that over $90 \%$ of $S$. coelicolor genes are conserved remarkably well in S. lividans. This observation is not surprising considering that even bacteria from other genera, namely Mycobacterium and Thermofibida, share a significant homology with genes in Streptomyces [46]. Punctuating this pattern of widespread conservation are 23 genomic islands comprising $>600$ genes that are conspicuously absent in S. lividans. Many of these islands exhibit hallmarks of horizontal gene transfer. Although it is generally believed that chromosome extremities are more prone to massive gene flux compared to the central "core" [2], we did not observe a higher occurrence of genomic islands near the chromosome ends (10 genomic islands in the "core" compared to eight in the chromosome "arms"). This could partly be explained for certain islands containing phage-related elements or integrative plasmids that target specific sequences rather than rely on genome instability for chromosome integration. There is at least one report of DNA amplifications in the "core" region of $S$. coelicolor indicating the chromosomal instabilities are not strictly limited to the extremities [47]. Many of these regions (eg. GI-1, GI-4, GI-5, Gi-15) harbor elements that could potentially benefit the host, asserting that they truly are "fitness islands". Such elements include antibiotic biosynthesis genes, drug resistance determinants, phage defense systems, and numerous metabolic functions.

It is notable that 14 of the 23 islands detected in this study were reported as putative laterally acquired regions through in silico analysis during sequencing of the $S$. coelicolor genome [5]. Half of those (7/14) were reported precisely as described here; for the rest, the exact boundaries of the islands do not match our results. This does not, however, imply that our results contradict previous observations. One likely possibility is that genes in any extended genomic island predicted by Bentley et al., although recently acquired, are well-conserved in S. lividans (probably acquired before speciation). Another possibility is that the apparent discrepancies are merely consequences of our strict criteria for absence that eliminated certain marginal cases. On the other hand, all boundary extensions (and new islands) reported in this work provide conclusive experimental evidence of recent gene transfer in those regions.

\section{Hints for genetic basis of suppressed antibiotic synthesis in S. lividans}

Over the years, identification of genetic determinants that suppress actinorhodin production in S. lividans has aroused considerable interest among researchers. Although numerous mutational and overexpression stud- ies have led to activation of antibiotic synthesis in S. lividans [48-52], to our knowledge, none have succeeded in identifying the evolutionary transformation that led to modulation of actinorhodin synthesis. Our results provide two important clues in this regard. One is the absence of a probable acyl-CoA dehydrogenase (SCO6938) in $S$. lividans, whose mutation in $S$. coelicolor caused a marked delay in onset of actinorhodin synthesis as well as aerial mycelium formation [38]. Acyl-CoA dehydrogenases are enzymes associated with breakdown of long-chain fatty acids and might potentially generate precursors for polyketide biosynthesis [53]. Of particular note is a mutant of an acyl-CoA synthetase (another enzyme involved in fatty acid catabolism) in S. coelicolor that was severely impaired in actinorhodin synthesis particularly in glucose containing medium [54] - an observation curiously reminiscent of the conditional suppression of actinorhodin biosynthesis in S. lividans in glucose containing medium. The other clue comes from the apparent absence of bldB (SCO5723) locus in S. lividans. At least two other paralogs of bldB (SCO3424 and SCO4542) are also known to exist in $S$. coelicolor chromosome, both of which are conserved in S. lividans. However, these paralogs do not substitute for bldB $-S$. coelicolor mutants of bldB fail to erect aerial hyphae and lack actinorhodin production; yet, S. lividans appears to be quite capable of forming aerial mycelia and spores. Despite the apparent absence of SCO5723 locus in S. lividans, we also noted that a S. lividans gene annotated as bldB (GenBank accession AF071232) has been cloned and expressed in E. coli [55]. A closer look revealed that this $S$. lividans bldB shares only $\sim 82 \%$ homology with SCO5723 and hence gave a weak hybridization signal - an observation further corroborated by the failure of stringent Southern hybridizations to reveal the presence of bldB in S. lividans [56]. Perhaps, this level of similarity is sufficient for most of the functional activity of BldB. Notably, all the conserved residues reported by Eccleston et al. [57] are present in both versions of the protein. Nonetheless, our data suggests that this ortholog in S. lividans is present at an alternate locus since an entire 17-gene $S$. coelicolor segment (Gi-15) including SCO5723 is absent in S. lividans. We note that the other bldB paralogs of $S$. coelicolor have a much lesser degree of homology with $S$. lividans bldB.

\section{Absence of certain endonucleases and proteases in $\mathbf{S}$. lividans}

Another question of particular interest in this comparative study is what genetic factors make S. lividans a preferred host for heterologous protein expression? As mentioned earlier, the two contributing factors in this regard are the absence of a strong restriction-modification system and diminished extracellular protease activity. The phage growth limitation (Pgl) system found in GI-4 contains elements required for DNA restriction. Although this 
explains the unstable $\phi \mathrm{C} 31$ lysogens of $S$. lividans, introduction of this system into $S$. lividans did not impair its ability to uptake methylated DNA [36]. Two other potential candidates - SCO4213 and SCO4631 (both annotated as hypothetical proteins) were identified in a scan for methyl-sensing restriction endonucleases. SCO4213 contains a signature Mrr_cat type II restriction enzyme domain while SCO4631 is similar to an E. coli methylcytosine-specific restriction enzyme with an $\mathrm{HNH}$ endonuclease domain. In addition, SCO2863 - a putative helicase, also contains an $h s d R$-like type I restriction endonuclease domain. Moreover, the absence of two DNAmethylases, SCO6844 and SCO6885 might explain why DNA obtained from $S$. lividans is readily transformable into other streptomycetes. The attenuated extracellular protease activity in S. lividans is, perhaps, explained by the absence of two secreted serine proteases - SCO5722 and SCO5731.

TTA codon modulated gene expression is a recent evolutionary incorporation

An interesting observation that we noted in our analysis is the unusually high frequency of the rare TTA codon containing genes designated as absent in S. lividans. About $30 \%(43 / 145)$ of such genes are absent in S. lividans - statistically a much higher frequency compared to only $8 \%$ of all genes absent. Considering that AT-rich segments frequently occur in bacterial phages and plasmids [58] and also the fact that occurrences of TTA codons are quite rare in a GC-rich organism like $S$. coelicolor, it is likely that many of these genes are of foreign origin. In fact, a recent report by Chater and Chandra postulated that over $80 \%$ of TTA-containing S. coelicolor genes were acquired through horizontal gene transfer [46]. They speculated that bldA (the sole tRNA that can efficiently translate TTA) might itself have been laterally acquired, and that TTA-modulated protein expression is a very recent evolutionary adaptation. Our experimental results are consistent with their hypothesis.

\section{Conclusion}

Despite the presence of over 600 genes in these S. coelicolor genomic islands, we found that over 93\% were not highly expressed under typical laboratory conditions analyzed in this study. This is probably due to the fact that these elements require specific environmental stimuli for activation. Experimental conditions covering a wider range of physiological conditions will need to be tested for this purpose. Another rather likely possibility is that our genomic tools were simply not sensitive enough to detect certain physiologically relevant gene expression levels. We also note that genomic islands present in S. lividans and absent in S. coelicolor could not be identified in this study. Notwithstanding these limitations, our work sheds light into possible genetic determinants contributing to pheno- typic differences between S. coelicolor and S. lividans. More importantly, it lays a strong foundation for identification of specific gene targets in the genetically well-characterized S. coelicolor to engineer it for industrial protein or secondary metabolite production processes.

\section{Methods \\ Strains and culture conditions}

Spores for S. coelicolor M145 and S. lividans TK21 were generated on Mannitol-Soy flour or R5 agar [4]. Cultures for genomic DNA preparation were performed in YEME medium with $0.5 \%$ glycine supplement at $30^{\circ} \mathrm{C}$ until early stationary phase.

\section{Genomic DNA extraction and labeling}

Genomic DNA (gDNA) extraction was carried out using Kirby mix procedure as described elsewhere [4]. About $500 \mu \mathrm{l}$ of $20 \mu \mathrm{g} / \mu \mathrm{l}$ gDNA was sonicated briefly for 30-40 $\mathrm{sec}$ for shearing them to $\sim 500$ bp average size (confirmed by gel electrophoresis). The DNA was then labeled with Label IT ${ }^{\circledast} \mathrm{Cy} 3$ or Cy5 Labeling Kit (Mirus Bio Corp., Madison, WI) according to suppliers instructions.

\section{Microarray hybridizations and image analysis}

Samples containing 500 ng each of Cy3 and Cy5 labeled gDNA from S. coelicolor M145 and S. lividans TK21, respectively were hybridized to a whole-genome $S$. coelicolor microarray as described previously [25]. Hybridizations were carried out in triplicate for $\sim 16 \mathrm{hr}$ at $50^{\circ} \mathrm{C}$; arrays were washed and scanned using ScanArray5000 (Perkin Elmer, Wellesley, MA). Images were analyzed using GenePix (Axon Instruments, Union City, CA) to obtain raw intensity data for each spot. The median fluorescence intensity from each spot was used for all subsequent analysis.

\section{Array normalization and data analysis}

Raw relative intensity values for each spot were first normalized by scaling one of the gDNA channel signal intensities by a normalization factor to set the total intensity from both channels as equal. $\log _{2}$ hybridization signal ratios were then calculated from normalized intensities as $\log _{2}\left[\mathrm{gDNA}_{\text {Sliv }} / \mathrm{gDNA}_{\text {Scoe }}\right]$ and values were averaged using the median from triplicate experiments. Presence of at least three consecutive (in chromosome order) $\log _{2}$ $\left[\mathrm{gDNA}_{\text {Sliv }} / \mathrm{gDNA}_{\text {Scoe }}\right]$ values less than its global mean minus one standard deviation was taken as evidence for absence (or divergence) of a contiguous genomic segment in S. lividans. Certain marginal cases were thereafter manually reassigned as present/absent in S. lividans based on overwhelming trends of neighboring genes. For visualization purposes, median intensity ratios from successive 19gene sliding windows were plotted as a function of genes in chromosome order. 
For transcriptome analysis, cDNA-gDNA based microarray data was normalized using quantile normalization [25].

All microarray data discussed here are available at Gene Expression Omnibus (GEO): Accession - GSE7167 (comparative genomics hybridizations) and GSE7172 (transcriptome data).

\section{Sequence analysis}

Regional GC variations in S. coelicolor genome were calculated using $z^{\prime}$ curve method proposed by Zhang and Zhang [59]. Briefly, $z_{n}$ was calculated at genomic location $n$ as:

$$
z_{n}=\left(\mathrm{A}_{n}+\mathrm{T}_{n}\right)-\left(\mathrm{G}_{n}+\mathrm{C}_{n}\right), n=0,1,2 \ldots \mathrm{N}
$$

where $\mathrm{A}_{n}, \mathrm{~T}_{n}, \mathrm{G}_{n}$ and $\mathrm{C}_{n}$ are the cumulative number of bases A, T, G and C occurring in a sequence from $1^{\text {st }}$ to $n^{\text {th }}$ base. To amplify the deviations of $z_{n}$ from its average trend, a linear least square fit: $z_{n}=k n$ is performed and $z_{n}{ }^{\prime}$ is calculated as

$$
z_{n}^{\prime}=z_{n}-k_{n}
$$

Codon Adaptation Index (CAI), a measure of utilization of "optimal codons" was calculated as described previously [29]. First, a relative adaptiveness factor for every codon $i$ coding for amino acid $j$ is estimated as:

$$
w_{i, j}=\frac{f_{i, j}(G)}{f_{\max , j}(G)}, \text { for all codons } i \text { and amino acids } j
$$

where $f_{i, j}(G)$ is the frequency of occurrence of codon $i$ among the set for amino acid $j$ across the entire genome $G$ and $f_{\max , j}(G)$ is the corresponding value for the most frequently used codon for amino acid $j$. The CAI for a gene $g$ is then calculated as

$$
\mathrm{CAI}_{g}=\prod_{i=1}^{N} w_{i}^{1 / N}
$$

where $N$ is the number of amino acids in gene $g$. The algorithms for both cumulative GC plot and CAI plot were implemented using Matlab 7.0 with bioinformatics toolbox.

\section{PCR verification of islands absent in S. lividans}

The absence of genomic islands in S. lividans were verified by PCR using primers flanking each island. Primers were chosen so as to fall in or outside of the DNA probe segment used in microarray to improve chances of amplification. The PCRs were conducted using GC-rich ${ }^{\mathrm{TM}}$ PCR system or Expand ${ }^{\text {Tм }}$ long template PCR system (Roche Applied Science, Indianapolis, IN) with 2\% DMSO. Suc- cessful amplification of a relatively small product from $S$. lividans indicates absence of the intervening genomic island in each case.

\section{Authors' contributions}

KPJ analyzed and interpreted comparative genomic hybridization data, performed sequence analysis, carried out PCR verifications, performed transcriptomic and proteomic experiments and drafted the manuscript. WL participated in preliminary data analysis including data normalization and interpretations. FG conceived of the study and carried out comparative genomic hybridizations. DHS and WSH participated in discussions, critical review of results presented here and assisted in manuscript preparation. All authors have read and approved the final manuscript.

\section{Additional material}

\section{Additional file 1}

PCR verification for genomic islands - primers used and gel images. Details of primers chosen for PCRs to verify the absence of genomic islands in S. lividans and gel electrophoresis images of PCR products in each case. Click here for file

[http://www.biomedcentral.com/content/supplementary/14712164-8-229-S1.pdf]

\section{Additional file 2}

Table of microarray data and GI designations of genes probed. Contains comparative genomic microarray hybridization ratios from three replicate experiments, designations of presence/absence in $\mathrm{S}$. lividans, GI designations, CAI values and maximum/average expression in transcriptome data for each gene.

Click here for file

[http://www.biomedcentral.com/content/supplementary/14712164-8-229-S2.csv]

\section{Acknowledgements}

This study was supported in part by a grant from the National Institutes of Health (GM55850). Bioinformatics support was provided by the University of Minnesota Supercomputing Institute.

\section{References}

I. Challis GL, Hopwood DA: Synergy and contingency as driving forces for the evolution of multiple secondary metabolite production by Streptomyces species. Proc Natl Acad Sci USA 2003, 100(Suppl 2): |4555-6I.

2. Choulet F, Aigle B, Gallois A, Mangenot S, Gerbaud C, Truong C, Francou FX, Fourrier C, Guerineau M, Decaris B, et al.: Evolution of the terminal regions of the Streptomyces linear chromosome. Mol Biol Evol 2006, 23:236I-9.

3. Leblond P, Decaris B: Unstable linear chromosomes: the case of Streptomyces. In Organization of the prokaryotic genome Edited by: Charlebois RL. ASM press, Washington DC; 1999:263-89.

4. Kieser T, Bibb M], Buttner MJ, Chater KF, Hopwood DA: Practical Streptomyces Genetics The John Innes Foundation, Norwich, UK; 2000.

5. Bentley SD, Chater KF, Cerdeno-Tarraga AM, Challis GL, Thomson NR, James KD, Harris DE, Quail MA, Kieser H, Harper D, et al.: Complete genome sequence of the model actinomycete Streptomyces coelicolor A3(2). Nature 2002, 417:141-7. 
6. Anne J, Van Mellaert L: Streptomyces lividans as host for heterologous protein production. FEMS Microbiol Lett 1993, I I 4:121-8.

7. Binnie C, Cossar JD, Stewart DI: Heterologous biopharmaceutical protein expression in Streptomyces. Trends Biotechnol 1997 I5:3|5-20.

8. Gilbert M, Morosoli R, Shareck F, Kluepfel D: Production and secretion of proteins by streptomycetes. Crit Rev Biotechnol 1995, 15:13-39.

9. MacNeil DJ: Characterization of a unique methyl-specific restriction system in Streptomyces avermitilis . J Bacteriol 1988 170:5607-12.

10. Fornwald JA, Donovan MJ, Gerber R, Keller J, Taylor DP, Arcuri EJ, Brawner ME: Soluble forms of the human T cell receptor CD4 are efficiently expressed by Streptomyces lividans. Biotechnology (N Y) 1993, I I:1031-6.

II. Pozidis C, Lammertyn E, Politou AS, Anne J, Tsiftsoglou AS, Sianidis G, Economou A: Protein secretion biotechnology using Streptomyces lividans : large-scale production of functional trimeric tumor necrosis factor alpha. Biotechnol Bioeng 200I, 72:6II-9.

12. Zhang Y, Wang WC, Li Y: Cloning, expression, and purification of soluble human interleukin-4 receptor in Streptomyces. Protein Expr Purif 2004, 36:139-45.

13. Hong B, Wu B, Li Y: Production of C-terminal amidated recombinant salmon calcitonin in Streptomyces lividans. App Biochem Biotechnol 2003, II 0:113-23.

14. Lara M, Servin-Gonzalez L, Singh M, Moreno C, Cohen I, Nimtz M, Espitia C: Expression, secretion, and glycosylation of the 45 and 47-kDa glycoprotein of Mycobacterium tuberculosis in Streptomyces lividans. Appl Environ Microbiol 2004, 70:679-85.

15. Leblond P, Redenbach M, Cullum J: Physical map of the Streptomyces lividans 66 genome and comparison with that of the related strain Streptomyces coelicolor A3(2). J Bacteriol 1993 175:3422-9.

16. Zhou X, He X, Li A, Lei F, Kieser T, Deng Z: Streptomyces coelicolor A3(2) lacks a genomic island present in the chromosome of Streptomyces lividans 66. Appl Environ Microbiol 2004, 70:7110-8.

17. Dorrell N, Hinchliffe SJ, Wren BW: Comparative phylogenomics of pathogenic bacteria by microarray analysis. Curr Opin Microbiol 2005, 8:620-6.

18. Ochman H, Santos SR: Exploring microbial microevolution with microarrays. Infect Genet Evol 2005, 5: 103-8.

19. Zhou D, Han Y, Song Y, Tong Z, Wang J, Guo Z, Pei D, Pang X, Zhai J, Li M, et al.: DNA microarray analysis of genome dynamics in Yersinia pestis : insights into bacterial genome microevolution and niche adaptation. J Bacteriol 2004, I 86:5 I 38-46.

20. Dziejman M, Balon E, Boyd D, Fraser CM, Heidelberg JF, Mekalanos $\mathrm{JJ}:$ Comparative genomic analysis of Vibrio cholerae : genes that correlate with cholera endemic and pandemic disease. Proc Natl Acad Sci USA 2002, 99: I556-6I.

21. Fitzgerald JR, Sturdevant DE, Mackie SM, Gill SR, Musser JM: Evolutionary genomics of Staphylococcus aureus : insights into the origin of methicillin-resistant strains and the toxic shock syndrome epidemic. Proc Natl Acad Sci USA 200I, 98:882I-6.

22. Brosch R, Pym AS, Gordon SV, Cole ST: The evolution of mycobacterial pathogenicity: clues from comparative genomics. Trends Microbiol 200I, 9:452-8.

23. Murray AE, Lies D, Li G, Nealson K, Zhou J, Tiedje JM: DNA/DNA hybridization to microarrays reveals gene-specific differences between closely related microbial genomes. Proc Natl Acad Sci USA 200I, 98:9853-8.

24. Weaver D, Karoonuthaisiri N, Tsai HH, Huang CH, Ho ML, Gai S, Patel KG, Huang J, Cohen SN, Hopwood DA, et al:: Genome plasticity in Streptomyces : identification of I Mb TIRs in the S. coelicolor A3(2) chromosome. Mol Microbiol 2004, 5 I : I 535-50.

25. Mehra S, Lian W, Jayapal KP, Charaniya SP, Sherman DH, Hu WS: A framework to analyze multiple time series data: a case study with Streptomyces coelicolor. J Ind Microbiol Biotechnol 2006, 33: I59-72.

26. Hacker J, Carniel E: Ecological fitness, genomic islands and bacterial pathogenicity. A Darwinian view of the evolution of microbes. EMBO Rep 200I, 2:376-8I.

27. Ochman H, Lerat E, Daubin V: Examining bacterial species under the specter of gene transfer and exchange. Proc Nat Acad Sci USA 2005, 102(SuppI I):6595-9.
28. Zhang R, Zhang CT: A systematic method to identify genomic islands and its applications in analyzing the genomes of Corynebacterium glutamicum and Vibrio vulnificus CMCP6 chromosome I. Bioinformatics 2004, 20:6I2-22.

29. Jansen R, Bussemaker HJ, Gerstein M: Revisiting the codon adaptation index from a whole-genome perspective: analyzing the relationship between gene expression and codon occurrence in yeast using a variety of models. Nucleic Acids Res 2003, 3I:2242-5I.

30. Chung HJ, Kim EJ, Suh B, Choi JH, Roe JH: Duplicate genes for Fecontaining superoxide dismutase in Streptomyces coelicolor A3(2). Gene 1999, 231:87-93.

3I. Akopiants K, Florova G, Li C, Reynolds KA: Multiple pathways for acetate assimilation in Streptomyces cinnamonensis. J Ind Microbiol Biotechnol 2006, 33: 14|-50.

32. Bunet R, Brock A, Rexer HU, Takano E: Identification of genes involved in siderophore transport in Streptomyces coelicolor A3(2). FEMS Microbiol Lett 2006, 262:57-64.

33. Miao V, Coeffet-Legal MF, Brian P, Brost R, Penn J, Whiting A, Martin S, Ford R, Parr I, Bouchard M, et al:: Daptomycin biosynthesis in Streptomyces roseosporus : cloning and analysis of the gene cluster and revision of peptide stereochemistry. Microbiology 2005, I 5 I: I507-23.

34. Egan S, Wiener P, Kallifidas D, Wellington EM: Transfer of streptomycin biosynthesis gene clusters within streptomycetes isolated from soil. Appl Environ Microbiol 1998, 64:506I-3.

35. Kendall K, Cullum J: Cloning and expression of an extracellularagarase from Streptomyces coelicolor A3(2) in Streptomyces lividans 66. Gene 1984, 29:3|5-21.

36. Sumby $P$, Smith MC: Genetics of the phage growth limitation (Pgl) system of Streptomyces coelicolor A3(2). Mol Microbiol 2002, 44:489-500.

37. Birch A, Leiser A, Robinson JA: Cloning, sequencing, and expression of the gene encoding methylmalonyl-coenzyme $A$ mutase from Streptomyces cinnamonensis . J Bacteriol 1993, I75:35II-9.

38. Gehring AM, Wang ST, Kearns DB, Storer NY, Losick R: Nove genes that influence development in Streptomyces coelicolor . J Bacteriol 2004, I 86:3570-7.

39. Romero NM, Parro V, Malpartida F, Mellado RP: Heterologous activation of the actinorhodin biosynthetic pathway in Streptomyces lividans . Nucleic Acids Res 1992, 20:2767-72.

40. Keijser BJ, van Wezel GP, Canters GW, Kieser T, Vijgenboom E: The ram -dependence of Streptomyces lividans differentiation is bypassed by copper. J Mol Microbiol Biotechnol 2000, 2:565-74

4I. Eccleston M, Ali RA, Seyler R, Westpheling J, Nodwell J: Structural and genetic analysis of the BIdB protein of Streptomyces coelicolor. J Bacteriol 2002, 184:4270-6.

42. Sumby P, Smith MC: Phase variation in the phage growth limitation system of Streptomyces coelicolor A3(2). J Bacteriol 2003, 185:4558-63.

43. Hesketh AR, Chandra G, Shaw AD, Rowland JJ, Kell DB, Bibb MJ, Chater KF: Primary and secondary metabolism, and posttranslational protein modifications, as portrayed by proteomic analysis of Streptomyces coelicolor. Mol Microbiol 2002, 46:917-32.

44. Manteca A, Mader U, Connolly BA, Sanchez J: A proteomic analysis of Streptomyces coelicolor programmed cell death. Proteomics 2006.

45. Pallen MJ: The ESAT-6/WXGI00 superfamily - and a new Gram-positive secretion system? Trends Microbiol 2002, 10:209-12.

46. Chater KF, Chandra G: The evolution of development in Streptomyces analysed by genome comparisons. FEMS Microbiol Rev 2006, 30:65I-72

47. Redenbach $M$, Kleinert $E$, Stoll $A$ : Identification of DNA amplifications near the center of the Streptomyces coelicolor MI45 chromosome. FEMS Microbiol Lett 2000, 191:123-9.

48. Butler MJ, Bruheim P, Jovetic S, Marinelli F, Postma PW, Bibb MJ: Engineering of primary carbon metabolism for improved antibiotic production in Streptomyces lividans . Appl Environ Microbiol 2002, 68:473I-9.

49. Butler MJ, Takano E, Bruheim P, Jovetic S, Marinelli F, Bibb MJ: Deletion of scbA enhances antibiotic production in Streptomyces lividans. Appl Microbiol Biotechnol 2003, 61:512-6. 
50. Hu H, Zhang Q, Ochi K: Activation of antibiotic biosynthesis by specified mutations in the rpoB gene (encoding the RNA polymerase beta subunit) of Streptomyces lividans. I Bacteriol 2002, | 84:3984-9|.

5I. Kim ES, Hong HJ, Choi CY, Cohen SN: Modulation of actinorhodin biosynthesis in Streptomyces lividans by glucose repression of afsR2 gene transcription. J Bacteriol 200I, 183:2। 98-203.

52. Sola-Landa A, Moura RS, Martin JF: The two-component PhoRPhoP system controls both primary metabolism and secondary metabolite biosynthesis in Streptomyces lividans. Proc Natl Acad Sci USA 2003, 100:6133-8.

53. Zhang $Y X$, Denoya CD, Skinner DD, Fedechko RW, McArthur HA, Morgenstern MR, Davies RA, Lobo S, Reynolds KA, Hutchinson CR: Genes encoding acyl-CoA dehydrogenase (AcdH) homologues from Streptomyces coelicolor and Streptomyces avermitilis provide insights into the metabolism of small branchedchain fatty acids and macrolide antibiotic production. Microbiology 1999, I45(Pt 9):2323-34.

54. Banchio C, Gramajo H: A stationary-phase acyl-coenzyme A synthetase of Streptomyces coelicolor A3(2) is necessary for the normal onset of antibiotic production. Appl Environ Microbiol 2002, 68:4240-6.

55. Mishig-Ochiriin T, Won HS, Lee CJ, Kang SO, Lee BJ: Biophysical and structural property of the putative DNA-binding protein, BIdB, from Streptomyces lividans . Biopolymers 2003, 69:343-50.

56. Harasym M, Zhang LH, Chater K, Piret J: The Streptomyces coelicolor A3(2) b/dB region contains at least two genes involved in morphological development. J Gen Microbiol 1990, 136:1543-50.

57. Eccleston M, Willems A, Beveridge A, Nodwell JR: Critical residues and novel effects of overexpression of the Streptomyces coelicolor developmental protein BIdB: evidence for a critical interacting partner. J Bacteriol 2006, 188:8| 89-95.

58. Rocha EP, Danchin A: Base composition bias might result from competition for metabolic resources. Trends Genet 2002, | 8:29|-4.

59. Zhang R, Zhang CT: Genomic islands in the Corynebacterium efficiens genome. Appl Environ Microbiol 2005, 7 1:3 126-30.

Publish with Bio Med Central and every scientist can read your work free of charge

"BioMed Central will be the most significant development for disseminating the results of biomedical research in our lifetime. "

Sir Paul Nurse, Cancer Research UK

Your research papers will be:

- available free of charge to the entire biomedical community

- peer reviewed and published immediately upon acceptance

- cited in PubMed and archived on PubMed Central

- yours - you keep the copyright

Submit your manuscript here:

http://www.biomedcentral.com/info/publishing_adv.asp
BioMedcentral 